\title{
Predicting Household Water Use Behaviour for Improved Hygiene Practices in Internet of Things Environment via Dynamic Behaviour Intervention Model
}

\author{
Yang $\mathrm{Fu}^{1}$, Wenyan $\mathrm{Wu}^{2 *}$ \\ ${ }^{1}$ Arts and Creative Technologies, Staffordshire University, College Road, Stoke-on-Trent, \\ Staffordshire, United Kingdom \\ ${ }^{2}$ Arts and Creative Technologies, Staffordshire University, College Road, Stoke-on-Trent, \\ Staffordshire, United Kingdom \\ *w.wu@staffs.ac.uk
}

\begin{abstract}
Recent advances in Internet of Things enabled technologies allow the intelligent sensor systems to effectively and efficiently observe and identify human behaviour in many applications, particularly in energy consumption and healthcare sectors. One typical case is that how to use Internet of Thing (IoT) technologies to understand human water use behaviour for improved and sustained hygiene practice. Traditionally, static behaviour intervention models are widely utilised to simulate behaviour intervention process over time. These static methods can predict targeted human behaviour reasonably well, but lack of capabilities on understanding and responding behaviour change process in IoT environments. In this paper, we proposed a dynamic behaviour intervention model for predicting household water user behaviour for improved hygiene practices. This model is based on Expanded Theory of Planned Behaviour (ETPB), and adopted Structure Equation Model approach and Control Engineering Concept. A case study of household water consumption model using ANN is utilised to evaluate intervention trend of proposed ETPB dynamic behaviour model with system parameter identification. The ETPB dynamic model has been proved to be effective for modelling human behaviour intervention process.
\end{abstract}

\section{Introduction}

There is some overwhelming evidence that changing human behaviour has significant application meaning in addressing many public health concerns, such as diet control, sexual risk-taking, cancer [1-3] and improved hygiene practices. Effective monitoring and predicting certain type of human behaviour is of importance to deliver high quality disease prevention, diagnosis and treatment. Regarding various type of healthcare related human behaviours, the human behaviour of access to safe water and sanitation has attracted a lot of attentions from academic and industrial, due to its serious consequence to infectious diseases and plague in the developing countries. Good hygiene practices can reduce the incidence of diarrhoea, parasitic infections, skin and eye disease.

However, understanding and predicting human water use behaviour for improved hygiene practice is a challenging task for managers, clinicians, and policy-makers who seek to translate clinical advice and knowledge into practice $[4,5,6]$. Traditionally, behavioural interventions based hygiene programmes have been delivered principally face-to-face, and this continues to be the overwhelmingly dominant method of delivery. This dominant behaviour intervention method by giving personal advice face-to-face is very costly, and it is hard to offer every user with all day access to personal guidance and managing all their problems. 
Recently, with the development of Internet of Things (IoT) enabled healthcare technologies, it allows the provision of information adapted to unique behaviour of an individual, and deliver to large numbers of people who need to be addressed with health promotion information $[7,8,9,10]$. IoT technologies allow the smart water systems to effectively and efficiently observe and identify human water use behaviours for improved hygiene practices. These hygiene behaviours include: the use of water for personal cleanliness, safe food preparation and storage, environmental cleanliness in and around the household, disposal of solid waste and waste water, etc.

Typically, traditional fixed behaviour interventions are widely used for modelling and predicting hygiene behaviours, with a feature that the intervention schedule is applied to all participants and not taking into account any of their individual characteristics. This paper will investigate a new online behaviour intervention in IoT environment enables offering tailored information to individuals across time. For examples, a computer-delivered online drug abuse prevention program might be varied somewhat depending on the individual characteristics (e.g. location, education and income etc.), and the individual reaction are various over time towards the target behaviour programme with different acceptance levels. In previous researches, behaviour change theory or model is always used as a foundation of intervention design. Quantitative reviews of internet-based interventions techniques included human behaviour theories and models, such as Theory of Planned Behaviour, The Health Belief Model and Stage of Change (Transtheoretical model) etc. [11-15]. Most of models applied for modelling behaviour change are only based on static data analysis such as structure equation model [13, 16, 17]. Even these static methods shown their capabilities for predicting targeted issue, however, they did not demonstrate enough understanding of human behaviour change process and knowledge of behaviour intervention modelling temporally.

In this paper, a dynamic behaviour intervention model for predicting household water user behaviour was proposed to improved hygiene practices. This model uses control engineering concept, introduction of time delay and system coefficients for intervention process. This dynamic modelling offers a method using fluid analogy to understand behaviour intervention process over time and insight of how to simulate complex psychological system dynamically with control engineering concept. Theoretical basis of our dynamic model is Expanded Theory of Planned Behaviour (ETPB) [18], which firstly be expressed as a mathematical represent with Structure Equation Model, and then postulated as fluid analogy to introduce dynamic system factors. Apart from model, in this paper, ETPB questionnaire has also been designed as a support for collecting behavioural data from online users and three kinds of parameters in dynamic system are identified. A case study concerned household water use behaviour was applied by using data collected in China. An ANN based water consumption model was presented to depict the differences of behaviour intervention 
trend and evaluate effectiveness of the dynamic behaviour model with three types of parameters identification.

The major contributions of this paper are: 1. Propose a dynamic behaviour intervention model for understanding and predicting behaviour intervention process; 2. Develop an ETPB data collection method within IoT environment. These contributions will enhance the understanding of household water use behaviour for improving urban household hygiene practices.

The rest of this paper is organised as follows. Section 2 summarises the different attempts that are made to simulate behaviour change process. In Section 3, describes the online behaviour change methodology in IoT environment which contains theoretical basis, data collection, behaviour modelling and exploratory simulation. The case study and model evaluation of the dynamic ETPB model is carried out in Section 4, whereas the conclusions and the future work are presented in Section 5.

\section{Related Work}

The internet based behaviour change research has been extensively researched previously. For example, Thomas L Webb al. etc. [15] reviewed 85 studies published coding frames for assessing use of theory and behaviour change models to promote online health behaviour change. Most commonly used method for conducting behaviour change are theories which support to delineate the key determinants of behaviour, followed by statistic methods to analysis the factor relationship or linkages. Models and Theories widely applied include Theory of reasoned action/planned behaviour (TPB), Transtheoretical model (TTM), Health belief Model (HBM) and Social cognitive model (SCT) etc. As a support technology for analysis of behaviour change model, statistics methods are accepted by researchers [20]. Multiple Linear Regression and Structure Equation Model (SEM) are the commonly used statistical techniques in behaviour science.

In [19], an attitude-behaviour theory testing research was conducted using standard multiple regression to illustrate TPB timeline and path diagrams. [22] systematically analysed the applied multiple regression/correlation for the behaviour sciences with behaviour data visualization, exploration and assumption checking methods. [23] utilized multiple binary logistic regression analyses to estimate the association between different personality characteristics (self-efficacy, self-identity etc.). However, even a lot of research used this method, as a static statistic approach, multiple linear regression shows few its capability to analysis complicated system, especially simulate system change over time. [24] presented a method by using SEM to analyse the mediating conceptual framework (physical activity and sedentary behaviour). Interestingly, statistic approach using SEM is still valuable to simulate behaviour change but shown less capability to simulate intervention system over time. Engineering control principles showed its applicability to areas in the behavioural science which involve dynamical systems, such as time-varying 
adaptive intervention [25]. Fundamental control engineering and adaptive intervention terminology such as dynamic system (refer to multivariate time-varying process), tailoring variable and process analysis could benefit to current behaviour change modelling research. Even [26, 27] have successfully simulated behaviour change by introducing control engineering concept such as time delay, resistance etc. and proved this cross-discipline method can benefit behaviour change simulation research somehow, however, none of them provide a comprehensive and generic framework for behaviour change data collection and dynamic process modelling. In addition, there is lack of performance evaluation of dynamic modelling and its effects on the result prediction performance.

In this paper, therefore, we create dynamic behaviour change process model that could support generating a comprehensive behaviour change methodology, evaluation of performance and targeted issue prediction.

\section{Proposed Methodology}

As the human behaviour change process is rather complex, there are many steps that are involved in this work. Fig. 1 illustrates the online behaviour change methodology in IoT environment which can conveniently be used dealing with such complex issue through behaviour data collection and data feedback flow. In this study, Expanded Theory of Planned Behaviour questionnaire (ETPB Questionnaire) based on the Theory of Planned Behaviour [2], is utilized for supporting our data collection progress. Data then be processed, using ETPB dynamic model to simulate the targeted issue behaviour intervention process. Moreover, for the feedback simulation result, we can use game mechanism in this methodology to guide users with persuasive strategies, and motivation system is also could be applied as a support to achieve the set goals. All these feedback strategies will benefit to increase online behaviour change project's readability. Additionally, in order to validate personal intervention, a well-designed follow-up data recollection is also essential.

Fig. 1. Methodology of online behaviour intervention system in IoT environment

\subsection{Expanded Theory of Planned Behaviour}

The theoretical basis of the behaviour change methodology is ETPB, which is a derivative theory according to the widely applied behaviour change theory TPB [2]. Despite its significance in psychological research and practice, TPB became more controversial about its sufficiency and the need for external variables which assist to the improvement of intention prediction (see examples in references [27, 28]). Research findings above have proposed additional predictors to improve the TPB perdition power in 
intention and behaviour [29, 30]. ETPB pointed out that past behaviour and self-efficacy as external constructs of TPB, have significant influences towards targeted behaviour [32, 33]. Theoretical framework of ETPB is displayed in Fig. 2. In ETPB theory, Intention is an indication of the readiness of a person to perform a given behaviour, and behaviour acts as an observable response with respect to a given target. Attitude towards the behaviour refers to the degree of outcomes for an action, which might be positive or negative. Past-behaviour, self-efficacy and Perceived Behaviour Control mean the past actions which could influent decision making for conducting a targeted behaviour, strength of one's belief to complete task and perception ability to perform a given behaviour respectively. Details for all ETPB variable definition and determinants are given in reference [28].

\section{Fig. 2. ETPB conceptual structure}

\subsection{Data Collection}

Considering the wide application of internet and interconnected objects from IOT such as mobile phone, tablet, PC and e-reader etc., data collection and sharing for this behaviour intervention methodology is not limited to sources of websites, but also should consider other personalised device applications. As an aspect of health psychology, some data of human behaviour change could be collected directly or indirectly from these interconnected objects through IoTs, such as past behaviour computation by analysis wake up record of particular sensor. Also, as IoTs environment could provide, P. Yang et al. argued that GPS, internet access, sensor network and sensor ID data is also suggested as behavioural change investigation supporting data in our study $[9,10,34,35]$.

ETPB questionnaire is designed to collect behaviour data/objective parameter based on the standard TPB questionnaire and contains questions covering attitudes, intentions, former behaviour, moral norms, descriptive norms, and self-efficacy variables. It is proposed by using seven points Likert scale form and answer setting from "1" (means strongly disagree/dislike) to "7" (means strongly agree/like). For example, Attitude is directly measured by two questions about what reporter thinks about the given targeted behaviour (good - bad, harmful- beneficial), and Subjective Norm (SN) is assessed directly with the perceived social pressure to perform or not to perform the behaviour etc. Table 1 lists the ETPB questionnaire contents.

Table 1 ETPB Questionnaire Variable Setting

\subsection{Behaviour Change Modelling}


This behaviour intervention methodology is to offer an understanding of behaviour intervention through behaviour intervention process modelling. We introduced control engineering concept to simulate behaviour intervention over time by giving SEM time delay and corresponding coefficient, so that the behaviour intervention modelling process will be expressed clearly through this method.

The relationship between human behaviour change parameters and behaviour change mechanic was investigated using a Structural Equation Model with latent variables. SEM is widely used in psychology and other social sciences, although its properties are not clear when the model structure is complicated. SEM in this research only concern path analysis with assumptions that independent variables have no measurement error and problem variables could be observed. The linkage between past behaviour and subjective norm has been ignored in the simulation process because there is no apparent link for those two factors shown in previous research. The path diagram and variable definitions in SEM of Expanded theory of Planned Behaviour (ETPB) are depicted in Fig. 3.

As classical SEM model only represents a static system and cannot express behaviour changing over time, to expand the ETPB SEM model with dynamic effects, we proposed a fluid analogy to postulate and parallel inventory management in supply chains. Each endogenous variable of ETPB structure equation model is represented as different inventory, as shown in Fig 4. Generally, behaviour intervention of ETPB could be expressed by the accumulation of different inventories according to mass conservation principle in Equation (1):

$$
\text { "Inventory" Accumulation = Fluid Inflow - Fluid Outflow }
$$

Fig. 3. ETPB model path analyses and variable definition

Fig. 4. Fluid analogy for ETPB model

To generate time and mass transaction description, three kinds of parameters were introduced: system inventory mass relation $\left(\beta_{i j}\right.$ and $\left.\gamma_{i j}\right)$, inventory transaction time delay $\left(\theta_{i}\right)$ and inventory capacity constant $\left(\tau_{i}\right)$. Specifically, $\theta_{i}$ is inventory time transport delay, $\tau_{i}$ is constant to capture the capacity of each inventory, $\xi_{i}$ represents the outside influences. $\beta_{i j}$ and $\gamma_{i j}$ are weight factors of matrices regression respectively. $\xi$ is a vector of disturbance variables which is expressed by zero-mean stochastic signal in the system. Relaying on Equation (1), inventory $\left(\eta_{\mathrm{i}}\right)$ accumulation equations of fluid analogy system are generated accordingly: 


$$
\begin{aligned}
& \eta_{1}=\xi_{1}(t) \\
& =\beta_{21} \eta_{1}\left(t-\theta_{1}\right)+\beta_{31} \eta_{1}\left(t-\theta_{2}\right)+\beta_{51} \eta_{1}\left(t-\theta_{3}\right)+ \\
& \left(1-\beta_{21}-\beta_{31}-\beta_{51}\right) \eta_{1}(t) \\
& \tau_{2} \frac{d \eta_{2}}{d t}=\beta_{21} \eta_{1}\left(t-\theta_{1}\right)+\gamma_{22} \xi_{2}\left(t-\theta_{4}\right)-\beta_{62} \eta_{2}\left(t-\theta_{8}\right)-\left(1-\beta_{62}\right) \eta_{2}(t)+\zeta_{2}(t) \\
& =\beta_{21} \eta_{1}\left(t-\theta_{1}\right)+\gamma_{22} \xi_{2}\left(t-\theta_{4}\right)-\beta_{62} \eta_{2}(t)+\zeta_{2}(t) \\
& \tau_{3} \frac{d \eta_{3}}{d t}=\beta_{31} \eta_{1}\left(t-\theta_{2}\right)+\gamma_{33} \xi_{3}\left(t-\theta_{5}\right)-\beta_{63} \eta_{3}\left(t-\theta_{9}\right)-\left(1-\beta_{63}\right) \eta_{3}(t)+\zeta_{3}(t) \\
& =\beta_{31} \eta_{1}\left(t-\theta_{2}\right)+\gamma_{33} \xi_{3}\left(t-\theta_{3}\right)-\beta_{63} \eta_{3}(t)+\zeta_{3}(t) \\
& \tau_{4} \frac{d \eta_{4}}{d t}=\gamma_{44} \xi_{4}\left(t-\theta_{6}\right)-\beta_{64} \eta_{4}\left(t-\theta_{10}\right)-\left(1-\beta_{64}\right) \eta_{4}(t)+\zeta_{4}(t) \\
& =\gamma_{44} \xi_{4}\left(t-\theta_{6}\right)-\beta_{64} \eta_{4}(t)+\zeta_{4}(t) \\
& \tau_{5} \frac{d \eta_{5}}{d t}=\beta_{51} \eta_{1}\left(t-\theta_{3}\right)+\gamma_{55} \xi_{5}\left(t-\theta_{7}\right)-\beta_{65} \eta_{5}\left(t-\theta_{11}\right)- \\
& \beta_{75} \eta_{4}\left(t-\theta_{13}\right)-\left(1-\beta_{65}-\beta_{75}\right) \eta_{5}(t)+\zeta_{5}(t) \\
& =\beta_{51} \eta_{1}\left(t-\theta_{3}\right)+\gamma_{55} \xi_{5}\left(t-\theta_{7}\right)-\eta_{5}(t)+\zeta_{5}(t) \\
& \tau_{6} \frac{d \eta_{6}}{d t}=\beta_{62} \eta_{2}\left(t-\theta_{8}\right)+\beta_{63} \eta_{3}\left(t-\theta_{9}\right)+\beta_{64} \eta_{4}\left(t-\theta_{10}\right)+ \\
& \beta_{65} \eta_{5}\left(t-\theta_{11}\right)-\beta_{76} \eta_{6}\left(t-\theta_{12}\right)-\left(1-\beta_{76}\right) \eta_{6}(t)+\zeta_{6}(t) \\
& =\beta_{62} \eta_{2}\left(t-\theta_{8}\right)+\beta_{63} \eta_{3}\left(t-\theta_{9}\right)+\beta_{64} \eta_{4}\left(t-\theta_{10}\right)+ \\
& \beta_{65} \eta_{5}\left(t-\theta_{11}\right)-\eta_{6}(t)+\zeta_{6}(t) \\
& \tau_{7} \frac{d \eta_{7}}{d t}=\beta_{76} \eta_{6}\left(t-\theta_{12}\right)+\beta_{75} \eta_{5}\left(t-\theta_{13}\right)-\eta_{-}(t)+\zeta_{4}(t)
\end{aligned}
$$

Where, according to SEM model, $\eta_{1}(t)=\xi_{1}(t), \quad \xi_{2}(t)=\mathrm{b}_{1}(t) \times e_{1}(t), \quad \xi_{3}(t)=\mathrm{n}_{1}(t) \times m_{1}(t)$, $\xi_{4}(t)=\mathrm{c}_{1}(t) \times p_{1}(t)$ and $\eta_{5}(t)=\xi_{5}(t)$. When $\frac{d \eta_{i}}{d t}=0$, the dynamic system came to steady state and Equations (6) - (8) reduce to SEM model. System inventory changes are psychically interpreted as "rate of flow", only have first order relation with timet . 


\subsection{Exploratory Behaviour Simulation}

For illustrate purpose, the ETPB dynamic model is shown with step change to $\xi_{1}$. Initial values is set as $b_{0}=3, e_{0}=3, n_{0}=3, m_{0}=3, c_{0}=3, p_{0}=3$ to stand for a typical individual with slightly negative attitude at the beginning of intervention project. Time delay $\theta_{1} \sim \theta_{7}=0$, two days' delay to intention to behaviour with $\theta_{8} \sim \theta_{13}=2$ were assumed for the intervention. Inflow resistances $\gamma_{i i}=1$, transfer resistances $\beta_{i j}=0.5$ and time constants (in days) are $\tau_{1}=\tau_{2}=\tau_{3}=1, \tau_{4}=2, \tau_{5}=4$, which means this individual can understanding all the intervention strategy and perform this targeted behaviour within a week. The initial values at $t=0$ for past behaviour, self-efficacy, subjective norm and $\mathrm{PBC}$ are $\xi_{1}(0)=\xi_{2}(0)=20, \xi_{4}(0)=\xi_{5}(0)=9$ respectively. Fig. 5 shows the behaviour change process in ETPB model with three different targeted attitudes: $b_{1}=4, e_{1}=4 ; b_{1}$ $=5, e_{1}=6 ; b_{1}=7, e_{1}=6$. The unit for time in this model is 'day' and no outside influence is assumed.

In Fig. 5, different targeted attitude could lead to different behaviour value. The larger $\xi_{1}$ value, the larger intention and behaviour value. Note that $\xi_{1}=b_{1} \times e_{1}$, then the separated value for $b_{1}$ and $e_{1}$ cannot decide how behaviour value changes in this dynamic system independently. As a detail analyses for other input variables in Fig. 6, the step response for different time delay are examined. Note that the larger $\tau_{\mathrm{i}}$ leads to slower dynamics, which means, the system has a longer shift to a steady state and intervention strategy chosen takes more time to achieve goal.

As the curve of behaviour change process in Fig 6 (c) exists two periods with stable output, the two step jumps mainly is caused by time delays of the input and transfer. To understand and adjust these time delays correctly will benefit the effects of behaviour intervention strategies. If the behaviour change process could be interrupted with reasonable stimulation, the goal of behaviour change can reach earlier. Then the model inputs with $b_{1}=7, e_{1}=6$ as initial values and is tested by three different time delay sets from low, medium and high time delays in the dynamic system $\left(\theta_{1} \sim \theta_{7}=0, \theta_{8} \sim \theta_{13}=2\right), \quad\left(\theta_{1} \sim \theta_{7}=1, \theta_{8} \sim \theta_{13}\right.$ $=3),\left(\theta_{1} \sim \theta_{7}=3, \theta_{8} \sim \theta_{13}=4\right)$. The results with different initial simulation variables are shown in Fig. 6 as below.

Fig. 5. Behaviour change curve with variable attitude input (time unite is "day")

a attitude curve

$\mathrm{b}$ intention curve

c behaviour change curve

Fig. 6. Behaviour change curve with variable time delay (time unite is "day") a $\theta_{1} \sim \theta_{7}=0, \theta_{8} \sim \theta_{13}=2$ 
b $\theta_{1} \sim \theta_{7}=1, \theta_{8} \sim \theta_{13}=3$

c $\theta_{1} \sim \theta_{7}=3, \theta_{8} \sim \theta_{13}=4$

\section{Case Study and Evaluation}

A case study concerned household water use behaviour was applied to evaluate intervention trend with three kind $s$ of parameter identification in this research. In this section, two models for depicting the differences of behaviour intervention trend and evaluating effectiveness of the dynamic ETPB model were present. The first model is an ANN (Artificial Neural Networks) based water consumption model, which concerned a questionnaire calculated behaviour (Questionnaire calculated behaviour value is a degree to identify the achievement of targeted goal) and other household water use drivers. The second model is ETPB dynamic model. These two models are compared in a given situation to predict typical urban apartment water use behaviour.

\subsection{Data Collection}

In this case, the targeted community is located in the town centre of Shenzhen, China. It was developed by Estate Company in 2002. This is a typical Chinese urban mature community with 1480 households and all the buildings are designed as seven-floor apartment. Residents from sampled community have easy access to public facilities, shopping zone and schools. Out of 200 apartment households sampled in this case in January, 2013, but only 128 apartments which had the monthly water consumption recorded and returned the completed questionnaire, with return rate of $64 \%$. Nine extreme cases were eliminated through a test of multivariate outlier's identification with a use of criterion Marhalanobis' distance $\mathrm{P}<0.001$. For the monthly water consumption record, it was provided by Water Utilities and collected by meters installed in each household of sampled community. Reliability of water consumption record was tested by Cronbach's alpha with a result of 0.782. Apart from ETPB variables and water consumption record, House Size, NOR (Number of Residents) and Education etc., as household water consumption drivers, are concerned in data collection procedure to generate ANN simulation results. Table 2 shows the data collected from sample area with analysing result.

Table 2 Data collection and reliability test results

\subsection{ANN Water Consumption Model}

The structure of ANN model in this study is the multiple-layer networks. This multi-layer networks consist of an input layer of value consisting of house size (HS), number of resident (NOR), month (M), 
education level (EL), income level (IL) and ETPB Questionnaire Calculated behaviour value (BV), two hidden layers of perceptron and an output lay for outputting the Water Consumption Data (WCD with a unit of Ton/month). Numbers of nodes in hidden layer is set as two. BV (Questionnaire Calculated Behaviour Value) is calculated according to findings of regression equation in literature about water saving behaviour change [36]. The behaviour calculated from ETPB questionnaire record is followed by Equation as below.

$$
B V=(\text { observed Intention *.69) }+(\text { observed } P B C * .27)
$$

The model development was limited to predict internal demand component especially for those houses which are apartments and this is one kind of the most common houses in Chinese main cities with public sharing garden in the community. Additionally, household leakage was not considered as this is highly inconsistent and uncertain, and the leakage prediction is related to a range of other variables not collected in the questionnaire of modelling.

The artificial neural network for household water consumption is expressed as follows:

$$
W C D=F\left(W F\left(W R+\psi_{h} I\right)+\psi_{0} I\right)
$$

Where WCD is the standardized water consumption data as output vector, $\mathrm{R}$ is the standardized input vector which consisting house size (HS), number of resident (NOR), month (M), education level (EL), income level (IL) and questionnaire calculated behaviour value (BV); $\mathrm{F}(\mathrm{x})$ is the sigmoid function; $W_{1}$ is the weight vector between input and hidden layer; $W_{2}$ is the weight vector between hidden and input layer; $\psi_{h}$ and $\psi_{o}$ are the threshold vector of hidden layer and output layer; $I$ is an unit matrix. 50 prediction data was compared with original data to test the accuracy of this ANN model. The accuracy of testing result in this model is shown in Fig. 7. The mean prediction error (\%) of ANN water consumption model between the original data and predication data is less than $0.8 \%$.

Fig. 7. Prediction error test of ANN water consumption model a Prediction result and its error b Prediction error in percentage

\subsection{ETPB Model Evaluation}

Model evaluation in this section is mainly discussed with two parts: evaluation of behaviour intervention trend and estimation of system parameters. To identify the effectiveness of dynamic ETPB model, we compared the results generated from ANN water consumption model with the results generated from ETPB behaviour change model by giving a particular setting of household, such as EL, NOR etc. to both models, the different household settings might lead to different water consumption trend, namely, different household water end use behaviour. 
In this case study, all inventory values are directly measured by ETPB questionnaire, inflow resistances of each inventory could be assumed as $\gamma_{22} \sim \gamma_{44}=1$. Under this assumption, inventory output transfer resistances could be interpreted by inventory coefficients. Mechanism for setting transfer resistances variables is referred to TPB parameter coefficients and regression equation analysis research in literature which offers a mega analysis of behaviour change parameters' correlation and regression [37], thus, transfer resistances are set as $\beta_{21}=0.3, \beta_{31}=0.2, \beta_{51}=0.4$. In order to present a typical reporter, initial mass input of each inventory is set by using mean variable value with $b_{0}=3, e_{0}=3, n_{0}=1, m_{0}=5, c_{0}=5, p_{0}=1$. Time constant $\tau_{i}$ is the mean rate of initial indirect measurement of inventory to direct measurement of inventory (ETPB questionnaire score), thus, time constant in this case is assumed as $\tau_{i}=1$. Three intervention strategy is simulated with dynamic expression of system time delay parameters $\left(\theta_{1} \sim \theta_{7}=0, \theta_{8} \sim \theta_{13}=2\right),\left(\theta_{1} \sim\right.$ $\left.\theta_{7}=1, \quad \theta_{8} \sim \theta_{13}=3\right), \quad\left(\theta_{1} \sim \theta_{7}=3, \theta_{8} \sim \theta_{13}=4\right) \quad$ (unit of delay is "month"), by which means, this intervention strategy could influent reporter's attitude, PBC, SN and Self-efficacy immediately. All intervention procedure will take 4 months to complete. Fig 8 (a) shown the ETPB behaviour intervention process simulation with variable intervention designs and outside influence (white noise signal $\varsigma_{i}=N(0,5)$ ).

The result of water consumption prediction from ANN is generated for behaviour intervention trend in comparison to ETPB dynamic model. ANN initial inputs are set as HS (House Size) = 2, NOR (Number of Resident) =3, EL (Education Level) =3, IL (Income Level) =2, which stands for a $87 \mathrm{~m} * \mathrm{~m}$ apartment with three residents older than 12 years old, education level for this reporter/representative (normally represent the landlord's education level) is bachelor degree and household annual income is $100 \mathrm{~K}-200 \mathrm{~K}$ RMBs.

The output of behaviour from ETPB should be interpreted as amount of water saved in this case. Because the BV and ETPB behaviour (amount of water saved) have first order relationship, therefore, if the increase of amount of water saved in Fig 8 (a) could match water consumption decrease trend in Fig 8 (b), we will assume ETPB dynamic model could generate right behaviour intervention trend. In Fig 8 (a), curve of ETPB model is with a steady start at the beginning and shows a rapid increase in the middle, followed by relatively steady trend at the end. Fig 8 (b) can generally match Fig 8 (a)'s trend with a rapid decrease of water consumption in the middle of curve, followed by a relatively steady statue at the end. However, for the beginning part of the intervention (trend of curve), ETPB model shown less capability to simulate the increase of amount of water saved at the early beginning in Fig 8 (a). That is because that the setting of dynamic system is with first order deviation in inventory equation which could not simulate inverse response. 
The evaluation result generated in Figure $8 \mathrm{a}$ and $8 \mathrm{~b}$ for this household water using case, shown that proposed ETPB model could effectively predict household behaviour intervention pattern by compared to an ANN household water consumption model. The trend of behaviour intervention pattern could match the trend of ANN water consumption result from the input zone [23, 43]. However, for the beginning of the household water use behavioural intervention process, these two models cannot match perfectly. To improve the prediction accuracy of proposed model, second order deviation dynamic system should be investigated in the further research.

Fig. 8. Behaviour Intervention of ETPB and ANN water consumption model a ETPB behaviour intervention with outside influence

b ANN behaviour intervention (one step change BV versus water consumption)

\section{Conclusion}

In this paper, an online behaviour intervention methodology was proposed in IoT environment for understanding the behaviour intervention and its relationship with targeted issue. This methodology contains data collection mechanism and modelling methods by simulating a psychological behavioural intervention conceptual model (ETPB). Contrastive evaluation results between ANN model and ETPB model from a real case study of household water end use is shown that this ETPB modelling method has successfully explored a way to simulate behaviour change and its behaviour intervention trend could generally match our ANN model.

The dynamic model in this paper can be used to simulate household water use behaviour under a particular intervention programme. The modelling method for understanding the behaviour intervention process has potentials to predict and improve hygiene practises, which also can also be applied to investigate any behaviour change such as energy saving, GHG control and water footprint research.

However, the limitation for this study is argued as follows. Firstly, the ETPB model cannot cover all the information of the behaviour change mechanism such as self-cognitive. It can be improved by further psychological research and other methods, like multivariate modelling method and time extrapolation method etc. for simulation comparison. Secondly, the intervention strategy in this dynamic ETPB model should also been identified with its strength of intervention and time delay for each inventory by using long term observation data. Thirdly, other stochastic technologies likes GRNN (Generalized Regression Neural Networks), CCNN (Cascade Correlation Neural Network) and FFNN (Feed Forward Neural Networks) are suggested to be applied in the generating prediction result. But there is still lacks of testing these kind of approach in this paper. Further research can be deployed in the above fields to overcome the limitations. 


\section{Acknowledgments}

The authors acknowledge the financial support of the European the Seventh Framework Program (FP7) WatERP (318603) and FP7 Marie Curie Actions-SmartWater (318985).

\section{References}

[1] Conner, M., Norman, P.:'Predicting and Changing Health Behaviour: Research and Practice with Social Cognition Models'(Open University Press, 3nd edn, 2015).

[2] Davis, R., Campbell, R., Hildon, Z., et al.:' Theories of behaviour and behaviour change across the social and behavioural sciences: a scoping review'. Health Psychol Rev, 2014, 8, pp.1-22.

[3] Michie, S., West, R., et al.:'ABC of Behaviour Change Theories' (Silverback Publishing, 1nd edn, 2014).

[4] Mark, Johnson., Carl, May.:'Promoting professional behaviour change in healthcare - what interventions work, and why? Protocol for a theory-led overview of systematic reviews', BMJ Open, 2015, 5, (9), pp. 1-10.

[5] J. Qi, P. Yang, et al.:'A survey of Physical Activity Monitoring and Assessment Using Internet of Things technology', the 2015 IEEE International Conference on Pervasive Intelligence and Computing, Liverpool, UK, October 2015, pp. 2353-2358.

[6] Z. Deng, P. Yang, et al.:'Life-logging data aggregation solution for interdisciplinary healthcare research and collaboration', the 2015 IEEE International Conference on Pervasive Intelligence and Computing, Liverpool, UK, October 2015, pp. 2315-2320.

[7] Alok, K., Sampada, S.:'Healthcare applications of the Internet of Things: A Review', International Journal of Computer Science and Information Technologies, 2014, 5(5), pp. 6229-6232.

[8] C, Chibelushi.:'An Overview and Concerns on IoT in Healthcare Applications: The Role of AI and Pattern Recongnition', International Journal of Information and Communication Technology Trends, 2014, 1, (1), pp. 39-52.

[9] P. Yang, W. Wu.:'Efficient Particle Filter Localisation Algorithm in Dense Passive RFID Tags Environment', IEEE Transactions on Industry Electronics, 2014, 61, (10), pp. 5641 - 5651.

[10] P. Yang, W. Wu, et al.:'Efficient Object Localisation Using Sparsely Distributed Passive RFID Tags', IEEE Transactions on Industry Electronics, 2013, 60, (12), pp. 5914-5924.

[11] Frances, G., Antje, L., John, P., et al.:'Why Are Health Care Interventions Delivered Over the Internet? A Systematic Review of the Published Literature', Journal of Medical Internet Research, 2006, 8, (2), e10.

[12] Su-I, Hou., Su-Anne, C., et al.:'Systematic literature review of Internet interventions across health behaviour', Health Psychology and Behaviour Medicine, 2014, 2, (1), pp. 455-481.

[13] Munro, S., Lewin, S., et al.: 'A review of health behaviour theories: how useful are these for developing interventions to promote long-term medication adherence for TB and HIV/AIDS?', Bmc Public Health, 2007, 7, (1), pp. 104.

[14] Chatterton, T.:'An introduction to Thinking about 'Energy Behaviour': a Multi Model Approach' (Department for Energy and Climate Change, 2011), pp. 9-15. 
[15] Thomas, W., Judith, J., et al.:'Using the Internet to Promote Health Behaviour Change: A Systematic Review and Meta-analysis of the Impact of Theoretical Basis, Use of Behaviour Change Techniques, and Mode of Delivery on Efficacy', Journal of Medical Internet Research, 2010, 12, (1), e4.

[16] Chris, F., Paschal, S., and Paul, N.:'Simulating behaviour change interventions based on the theory of planned behaviour: Impacts on intention and action', British Journal of Social Psychology, 2007, 46, (1), pp. 43-68.

[17] Lustria, A., Noar, M., et al.:' A meta-analysis of web-delivered tailored health behaviour change interventions'. Journal of Health Communication, 2013, 18, (9), pp. 1039-1069.

[18] Icek, A.:'The Theory of Planned Behaviour', Organization Behaviour and Human Decision Processes, 1991, 50, pp. 179-211.

[19] Bollen, A.:'Structural Equations with Latent Variables' (John Wiley \& Sons, 1nd edn, 1989).

[20] Andria, H., Carl, T., et al.:' Statistical synthesis of contextual knowledge to increase the effectiveness of theorybased behaviour change interventions'. J Health Serv Res Policy, 2011, 16, (3), pp. 167-171.

[21] Stephen, S.:' Testing attitude-behaviour theories using non-experimental data: An examination of some hidden assumptions'. European Review of Social Psychology, 2002, 13, pp. 293-323.

[22] Jacob, C., Patricia, C., et al.:' Applied Multiple Regression/Correlation Analysis for Behavioural Science' (Lawrence Erlabaum Associates, 3nd edn, 2002).

[23] Belmon LS., Middelweerd A., et al.:' Dutch Young Adults Ratings of Behavior Change Techniques Applied in Mobile Phone Apps to Promote Physical Activity: A Cross-Sectional Survey', JMIR mHealth uHealth, 2015, 3, (4), e103.

[24] Canfield, J.:'Models of Physical Activity and Sedentary Behavior Child and Family Studies'. PhD thesis, Syracuse University, 2012.

[25] Daniel, R., Michael, P. et al.:' Using engineering control principles to inform the design of adaptive interventions: A conceptual introduction', Drug and Alcohol Dependence, 2007, 88, pp. 31-40.

[26] Mohamed, H., Hajer, B., et al.:' A predictive model for recurrent consumption behaviour: An application on phone calls', Knowledge-Based Systems., 2014, 64, pp. 32-43.

[27] Yuwen, D., Daniel, R., et al.:' Hybrid Model Predictive Control for Optimizing Gestational Weight Gain Behavioural Intervention'. Porc. Am. Control. Conf, Washington, American, 2013, pp. 1970-1975.

[28] Yang, F., Wenyan, W.:'Behaviour interventions for water end use: An integrated model'. Proc. 20th Int. Conf. Automation and Computing, Cranfield, UK, Sept 2014, pp. 266-271.

[29] Mark, C., Christopher, A.:'Extending the Theory of Planned Behavior: A Review and Avenues for Further Research', Journal of Applied Social Psychology, 1999, 28, pp. 1429-1464.

[30] Norazlan, H., Abdul, M., and Mad, S.:'Extending the theory of planned behavior: Evidence of the arguments of its sufficiency'. International Journal of Humanities and Social Science, 2014, 4, (14), pp. 101-105.

[31] Hagger, S., Chatzisarantis, N., Biddle, J., et al.:'The influence of self-efficacy and past behaviour on the physical activity intentions of young people', Journal of Sports Sciences, 2001, 19, pp.711-725. 
[32] Martin, H., Nikos, C., et al.:'A The influence of self-efficacy and past behaviour on the physical activity intentions of young people', Journal of sports sciences, 2011, 19, (9), pp.711-725.

[33] Spinks, A., Fielding, K., et al., 'Water Demand Management Study: Baseline Survey of Household Water Use (Part A)' (Urban Water Security Research Alliance, 2011), pp. 16-32.

[34] P. Yang, M. Hanneghan, et al.:'Improving the Validity of Lifelogging Physical Activity Measures in an Internet of Things Environment', the 2015 IEEE International Conference on Pervasive Intelligence and Computing, Liverpool, UK, October 2015, pp. 2309-2314.

[35] E. G. Spanakis, D. Kafetzopoulos, P. Yang, et al.:' MyHealthAvatar: Personalised and empowerment health services through Internet of Things technologies', the 2014 EAI 4th International Conference on Wireless Mobile Communication and Healthcare, Athens, Greece, Nov 2014, pp. 331-334.

[36] Dilek, K., Sevilay, D.:'Examination of water saving behaviour within framework of Theory of Planned Behaviour', International Journal of Secondary Education, 2013, 1, (3), pp. 8-13.

[37] Rosemary, M., Mark, C., et al.:'Prospective prediction of health-related behaviours with the Theory of Planned Behaviour: a meta-analysis', Health Psychology Review, 2011, 5, (2), pp. 97-144. 